\title{
REMARKS ON CARISTI'S FIXED POINT THEOREM
}

\author{
M. A. KHAMSI
}

\begin{abstract}
In this work, we give a characterization of the existence of minimal elements in partially ordered sets in terms of fixed point of multivalued maps. This characterization shows that the assumptions in Caristi's fixed point theorem can, a priori, be weakened. Finally, we discuss Kirk's problem on an extension of Caristi's theorem and prove a new positive result which illustrates the weakening mentioned before.
\end{abstract}

\section{INTRODUCTION}

This work was motivated by a problem stated by Kirk [7] to improve the Caristi's fixed point theorem $[3,7]$. Recall that this theorem states that any map $T: M \rightarrow M$ has a fixed point provided that $M$ is complete and there exists a lower semi-continuous map $\phi$ mapping $M$ into the nonnegative numbers such that

$$
d(x, T x) \leq \phi(x)-\phi(T x)
$$

for every $x \in M$. This general fixed point theorem has found many applications in nonlinear analysis. It is shown, for example, that this theorem yields essentially all the known inwardness results [10] of geometric fixed point theory in Banach spaces. Recall that inwardness conditions are the ones which assert that, in some sense, points from the domain are mapped toward the domain. Possibly the weakest of the inwardness conditions, the Leray-Schauder boundary condition is the assumption that a map points $\mathrm{x}$ of $\partial M$ anywhere except to the outward part of the ray originating at some interior point of $M$ and passing through x. The proofs given to Caristi's result vary and use different techniques (see $[3,5$, $6,13])$. It is worth to mention that because of Caristi's result close connection

1991 Mathematics Subject Classification. Primary 06F30, 46B20, 47E10.

Key words and phrases. Partially ordered sets, minimal element, order preserving mappings, contraction and nonexpansive mappings, fixed point, multivalued mappings, subadditive functions. 
to the Ekeland's [8] variational principle, many authors refers to it as CaristiEkeland fixed point result. For more on Ekeland's variational principle and the equivalence between Caristi-Ekeland fixed point result and the completeness of metric spaces, the reader is advised to read [14].

In this work we prove a characterization to the existence of minimal elements in partially ordered sets in terms of fixed point of multivalued maps. Then we show how Caristi's theorem may be generalized. We will also discuss a proposed generalization by Kirk.

\section{Minimal Points and Fixed Point Property}

Let $A$ be an abstract set partially ordered by $\prec$. We will say that $a \in A$ is a minimal element of $A$ if and only if $b \prec a$ implies $b=a$. The concept of minimal element is crucial in the proofs given to Caristi's fixed point theorem.

Theorem 1. Let $(A, \prec)$ be a partially ordered set. Then the following statements are equivalent.

(1) A contains a minimal element,

(2) Any multivalued map $T$ defined on $A$ such that for any $x \in A$, there exists $y \in T x$ with $y \prec x$, has a fixed point, i.e there exists a in A such that $a \in T(a)$.

Proof. (1) $\Rightarrow$ (2) Obviously any minimal element is fixed by $T$. We complete the proof by showing that $(2) \Rightarrow(1)$. Assume that A fails to have a minimal element. Define the set valued map $T$ on $A$ by

$$
T(x)=\{y \in A ; \quad y \prec x \text { with } y \neq x\},
$$

for any $x \in A$. Clearly our assumption on $A$ implies that $T(x)$ is not empty for any $x \in A$. (2) will imply that $T$ has a fixed point $a \in A$. Contradiction with the definition of $T$, which completes the proof of Theorem 1.

Remark 1. Recall that Taskovic [15] showed that Zorn's lemma is equivalent to:

(TT) Let $\mathcal{F}$ be a family of selfmappings defined on a partially ordered set $A$ such that

$$
x \leq f(x)(\text { resp. } f(x) \leq x),
$$

for all $x \in A$ and all $f \in \mathcal{F}$. If each chain in $A$ has an upper bound (resp. lower bound), then the family $\mathcal{F}$ has a common fixed point. 
So, Theorem 1 is different from the above result since in our statement we consider the existence of minimal elements, which in general does not imply that any linearly ordered subset has a lower bound.

In the next result we discuss a common fixed point theorem. Let $(M, d)$ be a metric space and $\phi: M \rightarrow[0, \infty)$ be a map. Define the order $\prec_{\phi}($ see $[2,3,4])$ on $M$ by

$$
x \prec_{\phi} y \text { iff } d(x, y) \leq \phi(y)-\phi(x),
$$

for any $\mathrm{x}, \mathrm{y}$ in $M$. It is straightforward that $\left(M, \prec_{\phi}\right)$ is a partially ordered set. However it is not clear what are the minimal assumptions on $M$ and $\phi$ which oblige $M$ to have minimal elements. In particular, if $M$ is complete and $\phi$ is lower semi-continuous, then any decreasing chain in $\left(M, \prec_{\phi}\right)$ has a lower bound. Indeed, let $\left(x_{\alpha}\right)_{\alpha \in \Gamma}$ be a decreasing chain, then $\left(\phi\left(x_{\alpha}\right)\right)_{\alpha \in \Gamma}$ is a decreasing net of positive numbers. Let $\left(\alpha_{n}\right)$ be an increasing sequence of elements from $\Gamma$ such that

$$
\lim _{n \rightarrow \infty} \phi\left(x_{\alpha_{n}}\right)=\inf \left\{\phi\left(x_{\alpha}\right) ; \alpha \in \Gamma\right\} .
$$

Using the definition of $\prec_{\phi}$ one can easily show that $\left(x_{\alpha_{n}}\right)$ is Cauchy and therefore converges to $x \in M$. Finally, it is straightforward that $x \prec_{\phi} x_{\alpha_{n}}$ for all $n \geq 1$, which means that $x$ is a lower bound for $\left(x_{\alpha_{n}}\right)_{n \geq 1}$. In order to see that $x$ is also a lower bound for $\left(x_{\alpha}\right)_{\alpha \in \Gamma}$, let $\beta \in \Gamma$ such that $x_{\beta} \prec_{\phi} x_{\alpha_{n}}$ for all $n \geq 1$. Then we have $\phi\left(x_{\beta}\right) \leq \phi\left(x_{\alpha_{n}}\right)$ for all $n \geq 1$ which implies $\phi\left(x_{\beta}\right)=\inf \left\{\phi\left(x_{\alpha}\right) ; \alpha \in \Gamma\right\}$. Since $d\left(x_{\beta}, x_{\alpha_{n}}\right) \leq \phi\left(x_{\alpha_{n}}\right)-\phi\left(x_{\beta}\right)$, we get $\lim _{n \rightarrow \infty} x_{\alpha_{n}}=x_{\beta}$ which implies $x_{\beta}=x$. Therefore for any $\alpha \in \Gamma$, there exists $n \geq 1$ such that $x_{\alpha_{n}} \prec_{\phi} x_{\alpha}$ which implies $x \prec_{\phi} x_{\alpha}$, i.e. $x$ is a lower bound of $\left(x_{\alpha}\right)_{\alpha \in \Gamma}$. Zorn's lemma will therefore imply that $\left(M, \prec_{\phi}\right)$ has minimal elements.

Corollary 1. Let $(M, d)$ be a metric space and $\phi: M \rightarrow[0, \infty)$ be a map. Consider the partilly ordered set $\left(M, \prec_{\phi}\right)$. Assume that $a \in M$ is a minimal element. Then, any map $T: M \rightarrow M$ such that for all $x \in M$

$$
d(x, T x) \leq \phi(x)-\phi(T x),
$$

(i.e $\left.T x \prec_{\phi} x\right)$ fixes $a$, i.e $T a=a$. 
Remark 2. This corollary can be seen as a generalization of Caristi's result. Indeed, the regular assumptions made in Caristi's theorem imply that any linearly ordered subset $\left(\right.$ for $\prec_{\phi}$ ) has a lower bound, which is stronger than having a minimal element (see the remark following Theorem 1.).

Corollary 1 in fact contains implicitly a conclusion for the existence of a common fixed point. See [4] for a similar conclusion. Also it is worth to mention that the conclusion of Corollary 1 is similar to the famous result of Brodskii and Milman [1] who introduced the notion of normal structure of a convex set and proved that if $K$ is a convex, weakly-compact set with normal structure, then there is a common fixed point for the set of all surjective isometries of $K$. Note that this point is independent of the isometric mappings.

\section{KiRK's Problem}

In attempting to generalize Caristi's fixed point theorem, Kirk [7] has raised the problem whether a map $T: M \rightarrow M$ such that for all $x \in M$

$$
\eta(d(x, T x)) \leq \phi(x)-\phi(T x)
$$

for some positive function $\eta$, has a fixed point. In fact the original Kirk's question was stated when $\eta(t)=T^{p}$, for some $p>1$.

First let us give an example to answer Kirk's problem in the negative.

Example 1. Let $M=\left\{x_{n} ; n \geq 1\right\} \subset[0, \infty)$ defined by

$$
x_{n}=1+\frac{1}{2}+\frac{1}{3}+\ldots+\frac{1}{n},
$$

for all $n \geq 1$. Then $M$ is a closed subset of $[0, \infty)$ and therefore is complete. Define $T: M \rightarrow M$ by $T x_{n}=x_{n+1}$ for all $n \geq 1$. Then,

$$
d(x, T x)^{p}=\frac{1}{(n+1)^{p}}=\phi(x)-\phi(T x),
$$

where $\phi\left(x_{n}\right)=\sum_{i=n+1}^{\infty} \frac{1}{i^{p}}$, for all $n \geq 1$. It is easy to show that $\phi$ is lower semi-continuous. Furthermore one can also show that $T$ is nonexpansive, i.e $d(T x, T y) \leq d(x, y)$, for all $x, y \in M$. And it is clear that $T$ fails to have a fixed point. 
Though the above example gives a negative answer to Kirk's problem, some positive partial answers may also be found. Note that the order approach to the traditional Caristi's result is no longer possible. Indeed, if we define on the metric spaces $M$ the relation $x \prec y$ whenever $\eta(d(x, y)) \leq \phi(y)-\phi(x)$, then $\prec$ is reflexive and anti-symmetric. But it is not in general transitive. Of courser if $\eta$ is subadditive, i.e. $\eta(a+b) \leq \eta(a)+\eta(b)$ for any $a, b \in[0, \infty)$, then $\prec$ is transitive. This is the direction taken by the authors in [9]. We believe that the subadditivity of $\eta$ is very constraining. So one may wonder how to approach this general case when $\prec$ is not transitive and therefore $(M, \prec)$ is not a partial order. This is not the first time that the author has to deal with this kind of limitations. Indeed in [12] the authors dealt with a metric-like structure that fails the triangle inequality.

In what follows we assume that $\eta:[0, \infty) \rightarrow[0, \infty)$ is nondecreasing, continuous, such that there exist $c>0$ and $\delta_{0}>0$ such that for any $t \in\left[0, \delta_{0}\right]$ we have $\eta(t) \geq c t$. Because $\eta$ is continuous, then there exists $\varepsilon_{0}>0$ such that $\left.\eta^{-1}\left(\left[0, \varepsilon_{0}\right]\right)\right) \subset\left[0, \delta_{0}\right]$.

Under these assumptions we have the following result.

Theorem 2. Let $M$ be a complete metric space. Define the relation $\prec$ by

$$
x \prec y \Longleftrightarrow \eta(d(x, y)) \leq \phi(y)-\phi(x)
$$

where $\eta$ and $\phi$ satisfy all the above assumptions. Then $(M, \prec)$ has a minimal element $x_{*}$, i.e. if $x \prec x_{*}$ then we must have $x=x_{*}$.

Proof. Set $\phi_{0}=\inf \{\phi(x) ; x \in M\}$. For any $\varepsilon>0$, set

$$
M_{\varepsilon}=\left\{x \in M ; \phi(x) \leq \phi_{0}+\varepsilon\right\}
$$

Since $\phi$ is lower semi-continuous them $M_{\varepsilon}$ is a closed nonempty subset of $M$. Also note that if $x, y \in M_{\varepsilon}$ and $x \prec y$, then $\eta(d(x, y)) \leq \phi(y)-\phi(x)$ which implies

$$
\phi_{0} \leq \phi(x) \leq \phi(y) \leq \phi_{0}+\varepsilon
$$


Hence $\eta(d(x, y)) \leq \varepsilon$. Using $c, \varepsilon_{0}$, and $\delta_{0}$ associated with $\eta$ (as defined above), we get

$$
c d(x, y) \leq \eta(d(x, y)) \leq \phi(y)-\phi(x)
$$

for any $x, y \in M_{\varepsilon_{0}}$ with $x \prec y$. On $M_{\varepsilon_{0}}$ we define the new relation $\prec *$ by

$$
x \prec_{*} y \Longleftrightarrow d(x, y) \leq \frac{1}{c} \phi(y)-\frac{1}{c} \phi(x) .
$$

Clearly $\left(M_{\varepsilon_{0}}, \prec_{*}\right)$ is a partial order with all necessary assumptions to secure the existence of a minimal element $x_{*}$ for $\prec_{*}$. Let us see that $x_{*}$ is also a minimal element for the relation $\prec$ in $M$. Indeed let $x \in M$ such that $x \prec x_{*}$. Then we have $\eta\left(d\left(x, x_{*}\right)\right) \leq \phi\left(x_{*}\right)-\phi(x)$. In particular we have $\phi(x) \leq \phi\left(x_{*}\right)$ which implies $\phi(x) \leq \phi_{0}+\varepsilon_{0}$; i.e. $x \in M_{\varepsilon_{0}}$. As before we have $\eta\left(d\left(x, x_{*}\right)\right) \leq \varepsilon_{0}$ which implies

$$
c d\left(x, x_{*}\right) \leq \eta\left(d\left(x, x_{*}\right)\right) \leq \phi\left(x_{*}\right)-\phi(x)
$$

which implies $x \prec_{*} x_{*}$. Since $x_{*}$ is minimal in $\left(M_{\varepsilon_{0}}, \prec_{*}\right)$ we get $x=x_{*}$. This completes the proof of Theorem 2 .

The next result is a positive partial answer to Kirk's problem.

Theorem 3. Let $M$ be a complete metric space. Let $T: M \rightarrow M$ be a map such that for all $x \in M$

$$
\eta(d(x, T x)) \leq \phi(x)-\phi(T x)
$$

where the functions $\eta$ and $\phi$ satisfy the assumptions described above, then $T$ has a fixed point.

Proof. Define the relation $\prec$ as in Theorem 2. Obviously we have $T(x) \prec x$ for any $x \in M$. In particular if $x_{*}$ is a minimal element, then we must have $T\left(x_{*}\right)=x_{*}$.

This is an amazing result because the relation $\prec$ is not a partial order. Also the minimal point is fixed by any map $T$ so it is independent of the map.

Remark 3. Note that if $\eta$ is subadditive, then (see [11])

$$
\lim _{h \rightarrow 0} \frac{\eta(h)}{h}=\sup \left\{\frac{\eta(x)}{x} ; x>0\right\} \text {. }
$$


For the sake of completeness, let us give the proof of (SA). Since $\eta$ is subadditive, we have $\eta(n x) \leq n \eta(x)$, for any $x \geq 0$ and $n \geq 1$. Let $h$ and $x$ such that $0<$ $h<x$. Then there exists a unique $n(h) \geq 1$ such that $n(h) h<x \leq(n(h)+1) h$. Hence $\eta(x) \leq \eta((n(h)+1) h) \leq(n(h)+1) \eta(h)$ which implies

$$
\frac{\eta(x)}{x} \leq \frac{(n(h)+1) \eta(h)}{x} \leq \frac{(n(h)+1) \eta(h)}{n(h) h} .
$$

Since $\lim _{h \rightarrow 0} n(h)=\infty$, we get

$$
\frac{\eta(x)}{x} \leq \liminf _{h \rightarrow 0} \frac{\eta(h)}{h}
$$

Obviously this forces

$$
\limsup _{x \rightarrow 0} \frac{\eta(x)}{x} \leq \liminf _{h \rightarrow 0} \frac{\eta(h)}{h},
$$

which implies the existence of the desired limit. The identity (SA) follows easily from the inequality

$$
\frac{\eta(x)}{x} \leq \lim _{h \rightarrow 0} \frac{\eta(h)}{h}
$$

Clearly this identity will force $\lim _{h \rightarrow 0} \frac{\eta(h)}{h}>0$. Hence the constants $c>0$ and $\delta_{0}>0$ will exist such that for any $t \in\left[0, \delta_{0}\right]$ we have $\eta(t) \geq c t$.

A multivalued version of Theorem 4 may be obtained.

Theorem 4. Let $M$ be a complete metric space. Let $T: M \rightarrow \mathcal{P}(M)$ be a multivalued map such that $T(x)$ is not empty and for all $x \in M$ there exists $y \in T(x)$ such that

$$
\eta(d(x, y)) \leq \phi(x)-\phi(y)
$$

where the functions $\eta$ and $\phi$ satisfy the assumptions described above, then $T$ has a fixed point, i.e. there exists $x \in M$ such that $x \in T(x)$.

Proof. Define the relation $\prec$ as in Theorem 2. Obviously we have for any $x \in M$ there exists $y \in T(x)$ such that $y \prec x$. In particular if $x_{*}$ is a minimal element for $(M, \prec)$, then we must have $x_{*}=y$, for any $y \in T\left(x_{*}\right)$ such that $y \prec x_{*}$. Therefore we have $x_{*} \in T\left(x_{*}\right)$. 


\section{REFERENCES}

[1] M.S. Brodskii, D.P. Milman, On the center of a convex set, Dokl. Akad. Nauk SSSR 59 (1948), 838-840. (Russian)

[2] A. Brondsted, On a lemma of Bishop and Phelps, Pacific J. Math. Volume 55, Number 2 (1974), 335-341.

[3] A. Brondsted, Fixed point and partial orders,Proc. Amer. Math. Soc. 60(1976),365-366.

[4] A. Brondsted, Common fixed points and partial orders, Proc. Amer. Math. Soc. $77(1979), 365-368$.

[5] F. E. Browder, On a theorem of Caristi and Kirk, Proc. Seminar on Fixed Point Theory and Its Applications, Dalhousie University, June 1975, Academic Press, 23-27.

[6] J. Caristi, Fixed point theorems for mappings satisfying inwardness conditions, Trans. Amer. Math. Soc. 215(1976),241-251.

[7] J. Caristi, Fixed point theory and inwardness conditions, Applied Nonlinear Analysis,(1979),479-483.

[8] I. Ekeland, Sur les problemes variationnels, Comptes Rendus Acad. Sci. Paris, 275(1972),1057-1059.

[9] Y. Feng, S. Liu, Fixed point theorems for multi-valued ontractive mappings and multivalued Caristi type mappings, Journal of Mathematical Analysis and Applications, vol. 317, no. 1, pp. 103112, 2006.

[10] B. Halpern and G. Bergman, A fixed point theorem for inward and outward maps, Trans. Amer. Math. Soc. 130(1968),353-358.

[11] E. Hille and R.S. Phillips, Functional analysis and semi-groups, AMS, Colloquium Publications Vol.31, Providence, Rhode Island, 1957.

[12] M.A. Khamsi, W.K. Kozlowski, S. Reich, Fixed point theory in Modular function spaces, Nonlinear Analysis, Vol. 14(1990), 935-953.

[13] W. A. Kirk and J. Caristi, Mapping Theorems in Metric and Banach Spaces, Bull. L'Acad. Polon. Sci. 25(1975),891-894.

[14] F. Sullivan, A Characterization of Complete Metric Spaces, Proc. Amer. Math. Sot., 85(1981), 345-346.

[15] M. R. Taskovic, On an equivalent of the axiom of choice and its applications, Math. Japonica 31, No.6(1986),979-991.

Department of Mathematical Sciences, University of Texas at El Paso, El PASO, TX 79968-0514

E-mail address: mohamed@math.utep.edu 